SCIENTIFIC REPORT

\title{
Optical coherence tomography of the retinal nerve fibre layer in mild papilloedema and pseudopapilloedema
}

\author{
E Z Karam, T R Hedges
}

Br J Ophthalmol 2005;89:294-298. doi: 10.1136/bjo.2004.049486

Aims: To determine the degree to which optical coherence tomography (OCT) can distinguish differences in retinal nerve fibre layer (RNFL) thickness between eyes with mild papilloedema, pseudopapilloedema, and normal findings. Methods: 13 patients with mild papilloedema, 11 patients with congenitally crowded optic nerves, and 17 normal subjects underwent neuro-ophthalmic examination, automated visual field testing, and fundus photography. Spinal fluid pressure measurements were obtained in a subgroup of five patients with pseudopapilloedema and 11 patients with mild papilloedema. Circular OCT scans using a diameter of $3.38 \mathrm{~mm}$ surrounding the optic disc were performed in each eye of patients and subjects. Fundus photographs were analysed by two observers who diagnosed crowding or papilloedema and graded amounts of swelling. Findings were assessed by descriptive statistics and variance analysis. Results: RNFL thickness was greater in the superior and inferior quadrants and showed a high degree of correlation between each group of patients and subjects. A statistically significant difference was found in mean RNFL thickness between both groups of patients with optic disc swelling and normal subjects. However, there was not a statistical difference in mean nerve fibre layer thickness between patients with papilloedema and those with congenitally crowded optic nerves.

Conclusions: OCT demonstrates measurable differences in nerve fibre layer thickness between normal subjects and patients with either papilloedema or pseudopapilloedema. However, OCT does not appear to differentiate between those individuals with congenitally crowded optic nerves and those with mild papilloedema caused by increased intracranial pressure.

$M$ ild papilloedema caused by increased intracranial pressure may be difficult to distinguish by clinical observation alone from pseudopapilloedema caused by anomalous, congenital elevation of the optic disc. This distinction is critical because of the expense and invasiveness of some of the investigations, which may be undertaken to rule out causes of increased intracranial pressure in such patients. Several different techniques have been employed to distinguish pathological optic disc swelling from pseudopapilloedema with limited results. ${ }^{1-4}$

Optical coherence tomography (OCT) is a new technique, which can measure retinal nerve fibre layer (RNFL) thickness with spatial resolution of $10 \mathrm{~nm}$ or less. ${ }^{5-7}$ We sought to determine if OCT is an effective tool in the differential diagnosis of mild papilloedema and pseudopapilloedema by comparing groups of patients with both entities to normal control subjects.

\section{PATIENTS AND METHODS}

Thirty two patients with either crowded optic nerves or papilloedema (most of which was considered to be mild) were initially chosen for this study. All patients underwent neuro-ophthalmic examination, automated visual field testing (Humphrey 30-2, Allergan, Irvine, CA, USA), as well as colour and red free fundus photography. Additional neuroradiological studies and cerebrospinal fluid pressure measurements were obtained in 16 of these individuals.

Seventeen normal subjects were recruited. All had best corrected Snellen visual acuities of better than 20/20, refractive errors between +1.00 and -5.25 dioptres, normal Humphrey visual fields (no clusters of three or more adjacent points depressed more than $5 \mathrm{~dB}$ or two points depressed more than $9 \mathrm{~dB}$ ), intraocular pressure of $20 \mathrm{~mm} \mathrm{Hg}$ or lower, and no anomalous changes (for example, cup to disc ratio of less than 0.5, myelinated nerve fibres, abnormal vascular pattern) within the optic nerve heads. Other exclusion criteria for both patients and normal subjects included previous retinal or neurological disease (other than idiopathic intracranial hypertension or subdural/subarachnoid haemorrhage), the presence of optic disc drusen visible by ophthalmoscopy or OCT, atrophic papilloedema, glaucoma, any form of ocular surgery or laser therapy, or media opacities.

Red free photographs as well as colour photographs of all 32 patients and 17 control subjects were reviewed by two observers, one with over 20 years of experience in evaluating optic discs and the other with 5 years of experience. The photographs were mixed and shown randomly (fig 1) The observers were masked as to patient histories. Each observer was asked to identify each optic disc as normal, congenitally crowded, or papilloedematous. They were asked to identify optic discs with visible drusen. In addition, optic disc swelling was graded using a modification of the Frisen criteria for optic disc swelling ${ }^{6}$ as follows.

- Normal: diameter of optic disc cup between 10 and $50 \%$ of the overall optic disc diameter, no blurring of optic disc borders for $360^{\circ}$, and visibility of normal nerve fibres in all four quadrants.

- Stage 0: blurring of the superior and inferior region of the optic disc margin with visible nasal and temporal optic disc margins.

- Stage 1: additional nasal blurring with or without haemorrhage and a peripapillary halo.

- Stage 2: additional blurring nasally and temporally with or without haemorrhage and with or without retinochoroidal folds.

- Stage 3 (moderate papilloedema): blurring of all four quadrants of the disc margin with total obscuration of major vessel segments by swollen axons.

Abbreviations: OCT, optical coherence tomography; RNFL, retinal nerve fibre layer 

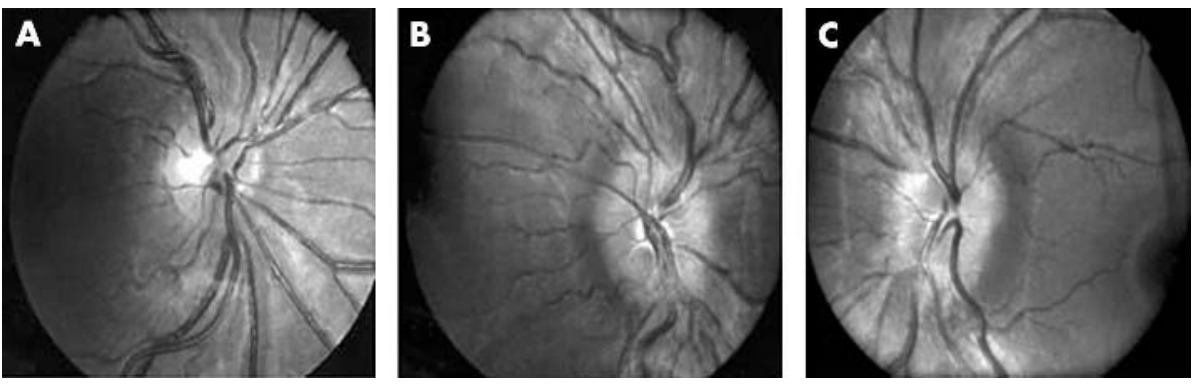

Figure 1 Red free fundus photographs. (A) Normal, (B) congenitally crowded, (C) mild papilloedema.

- Stage 4: obvious, well established papilloedema with haemorrhages and exudates.

Among the patients with papilloedema and pseudopapilloedema, three were eliminated because of the presence of visible optic disc drusen, and five were eliminated because they were classified as having more than moderate papilloedema (stage 3 or 4 ) by the Frisen criteria. This left 24 patients with either mild papilloedema or congenitally crowded optic discs.

Of the 24 normal subjects, three were rejected because the optic nerve heads were thought to be congenitally crowded. Four more were rejected because of myopia greater than -5.25 dioptres or because adequate photographs or examinations were not available. This left a final control group of 17 subjects ( 34 eyes).

Of the patients determined to have papilloedema, all but two had lumbar punctures showing intracranial pressure of more than $200 \mathrm{~mm} \mathrm{H}_{2} \mathrm{O}$. They also had findings consistent with idiopathic intracranial hypertension including headaches or double vision from sixth nerve palsy. All had normal magnetic resonance images. All but two were obese young females. Of the other two patients with papilloedema who did not have spinal fluid pressure measurements, one had a subarachnoid haemorrhage following trauma, and another had a subdural haematoma. In both, mild papilloedema resolved after treatment.

Of the 10 patients determined to have pseudopapilloedema, five had spinal fluid pressure measurements of less than $200 \mathrm{~mm} \mathrm{H}_{2} \mathrm{O}$. The other five had no signs or risk factors for increased intracranial pressure, and the appearance of their optic nerves remained stable during follow up for at least 1 year.

Patient ages ranged from 6 to 35 years (average 21.1 years), and normal subject ages ranged from 8 to 35 years (average 21.5 years).

OCT techniques and instrumentation have been described in detail elsewhere. ${ }^{5-8}$ The instrument employed in this study was the prototype OCT, equipped with a fibreoptic integrated Michelson interferometer with a short coherence length, super luminescent, near infrared diode source. The fibreoptic probe module was coupled to a slit lamp biomicroscope and a 78 dioptre lens (Volk, Mentor, OH, USA) to provide indirect imaging. The slit lamp was oriented by a trained OCT technician who was able to observe the fundus on a monitor. Circular scans were centred on the optic disc and performed at a circular diameter of $3.37 \mathrm{~mm}$ using internal fixation. A circle diameter of $3.37 \mathrm{~mm}$ was chosen because it avoided overlap with the optic disc, but was close enough to the disc for optimum comparison of RNFL thickness to baseline measurements. Using cross correlation scan registration, images were corrected for artefacts caused by involuntary subject movements. Scans were $32 \mathrm{~mm}$ deep, 100 pixels wide, and $110 \mu \mathrm{m}$ apart. Scanning time was 2.5 seconds with full width/half maximum with ranging resolution of $10 \mu \mathrm{m} .{ }^{78}$ No correction for refraction was made because the range of refractive error was from +1.00 to -5.25 dioptres. A digital filter was applied to smooth the tomogram and reduce image speckle noise. RNFL thickness was quantified by an image processing computer program which identified the first bright reflection at the vitreoretinal interface as the anterior boarder of the RNFL and the posterior border as a less reflective layer. ${ }^{8}$ RNFL thickness was reported as averages for each clock hour and as averages for each quadrant (superior, nasal, temporal, and inferior) (fig 2).

\section{RESULTS}

\section{Qualitative analysis of optic disc photographs}

In addition to selecting or rejecting patients with optic nerves containing drusen or other pathology, or rejecting patients with moderate to severe papilloedema, the masked observers classified all photographs as normal, crowded, or papilloedematous. There was agreement in $68.27 \%$ of the eyes (71 photographs) and disagreement in 31.73\% (33 photographs). Agreement by stages of optic disc swelling was 61.54\% (64 photographs). Although not documented in all cases, no
A

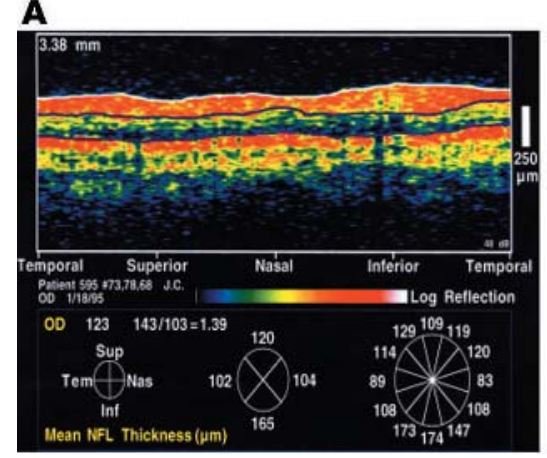

B

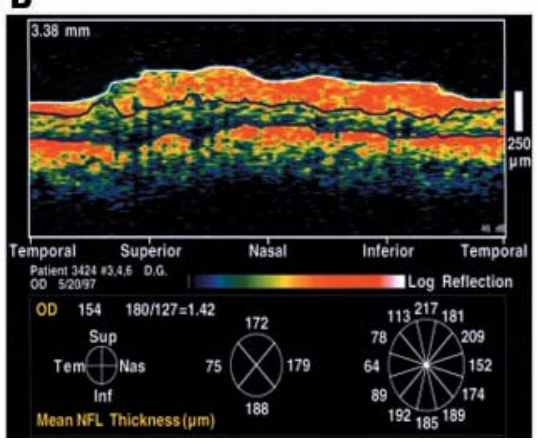

c

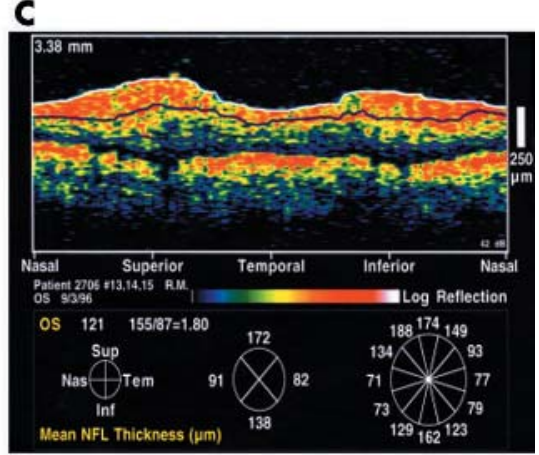

Figure 2 OCTs from the same eyes shown in figure 1. (A) Normal, (B) congenitally crowded, (C) mild papilloedema. 

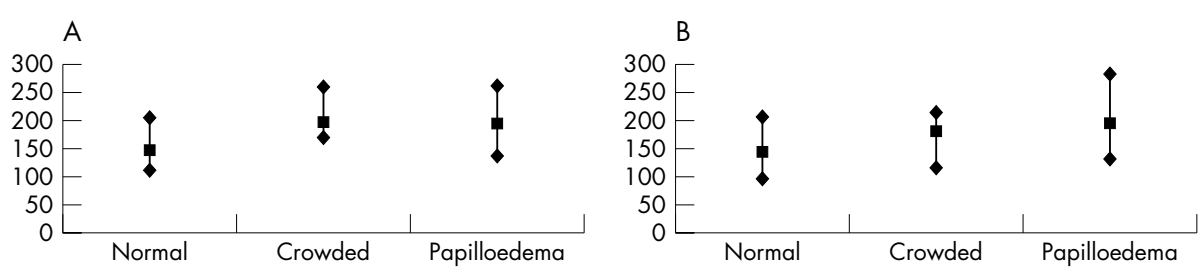

Figure 3 Distribution of nerve fibre layer thickness, average in all four quadrants in patients with known intracranial pressure. (A) Superior quadrant, (B) inferior quadrant, (C) nasal quadrant, (D) temporal Crowded Papilloedema
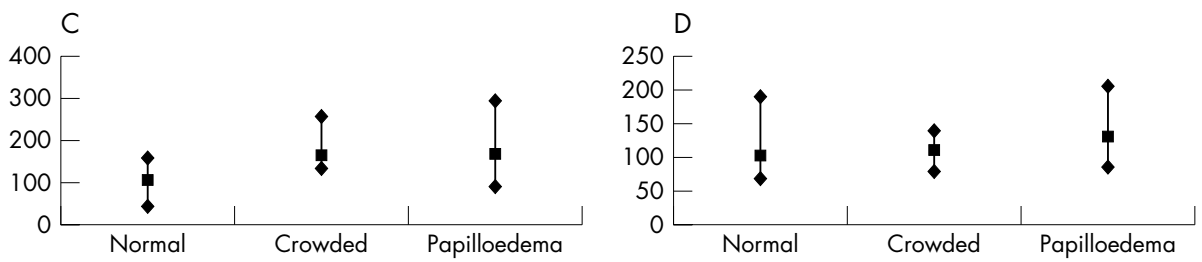

patient with papilloedema or pseudopapilloedema was noted to have spontaneous venous pulsation.

Most of the differences were observed in the differential diagnosis of crowded versus papilloedematous optic nerves. The more experienced observer tended to identify papilloedematous optic nerve heads as congenitally crowded, whereas the less experienced observer tended to identify crowded optic nerves as papilloedematous.

\section{Comparison of OCTs from normal subjects and patients with known intracranial pressure}

OCTs from five patients ( 10 eyes) with congenitally crowded nerves and normal intracranial pressures, and 11 patients (22 eyes) with mild papilloedema and increased intracranial pressures were compared to 17 control subjects (34 eyes). Eyes from both groups of patients had OCT measurements of RNFL thickness higher than control subject eyes. There was a statistical difference between true papilloedema and controls in all quadrants. However, eyes with crowded optic nerves showed significant differences primarily in the superior and nasal quadrants when compared to controls (see table 1).

Eyes with papilloedema and those with crowded optic nerves did not show statistically significant differences when compared quadrant by quadrant, and superior and nasal quadrants appeared to have closer values with a difference in the mean of 1.9 and 2.3 respectively (fig 3 ). The distribution of RNFL thickness by quadrant was the same in all patient groups in that RNFL thickness superiorly was greater than inferiorly, which in turn was greater than nasally and temporally. There was a broader range of thicknesses in all quadrants in the papilloedema group (fig 3 ).

\section{Comparison of OCTs from all patients with papilledema, all patients with crowded optic nerves and normals}

OCTs from all 13 patients with presumed or documented increased intracranial pressure, all 11 patients with presumed crowding of the optic nerves, and 17 normal control subjects were compared. Eyes with either papilloedema or congenitally crowded nerves showed higher RNFL thickness values than normal in all four quadrants. Eyes with papilloedema had statistically significant differences when compared to normals in all four quadrants (see table 2). The eyes with crowded optic nerves showed statistically significant differences when compared to normals in RNFL thicknesses in all quadrants except for the temporal quadrant. In addition, statistically significant differences were found between eyes with crowded nerves and those with papilloedema in both inferior as well as temporal quadrants. A significant overlap in the RNFL values between papilloedema and crowded nerves was observed in all quadrants. There was overlap between normal nerves and crowded nerves in the inferior and temporal quadrants. Similar overlap was noted in the nasal and temporal quadrants when papilloedema patients were compared to normals (fig 4).

Table 1 Student-Newman-Keuls comparisons of nerve fibre layer thickness by quadrant in normal, crowding, and papilloedema patients with known intracranial pressure

\begin{tabular}{|c|c|c|c|c|c|}
\hline Comparison & MD & SE & $p$ & $q$ & $\mathrm{p}<0.05$ \\
\hline \multicolumn{6}{|l|}{ Superior } \\
\hline $\mathrm{C} v \mathrm{~N}$ & 48.2 & 6.477 & 3 & 7.441 & Yes \\
\hline$C \vee P$ & 1.9 & 6.867 & 2 & 0.277 & No \\
\hline $\begin{array}{l}P \vee N \\
\text { Inferior }\end{array}$ & 46.3 & 4.927 & 2 & 9.398 & Yes \\
\hline$P \vee N$ & 50.8 & 6.458 & 3 & 7.867 & Yes \\
\hline$P \vee C$ & 14.3 & 9.001 & 2 & 1.589 & No \\
\hline $\begin{array}{l}C \vee N \\
\text { Nasal }\end{array}$ & 36.5 & 8.49 & 2 & 4.299 & No \\
\hline$P \vee N$ & 60.2 & 7.544 & 3 & 7.98 & Yes \\
\hline$P \vee C$ & 2.3 & 10.51 & 2 & 0.219 & No \\
\hline $\begin{array}{l}\mathrm{C} v \mathrm{~N} \\
\text { Temporal }\end{array}$ & 57.9 & 9.918 & 2 & 5.838 & Yes \\
\hline$P \vee N$ & 29.6 & 6.09 & 3 & 4.861 & Yes \\
\hline$P \vee C$ & 20.1 & 8.488 & 2 & 2.368 & No \\
\hline$C \vee N$ & 9.5 & 8.006 & 2 & 1.187 & No \\
\hline
\end{tabular}

C, crowded; N, normal; $P$, papilloedema; $M D$, mean deviation; $S E$, standard error Degrees of freedom: 63. 

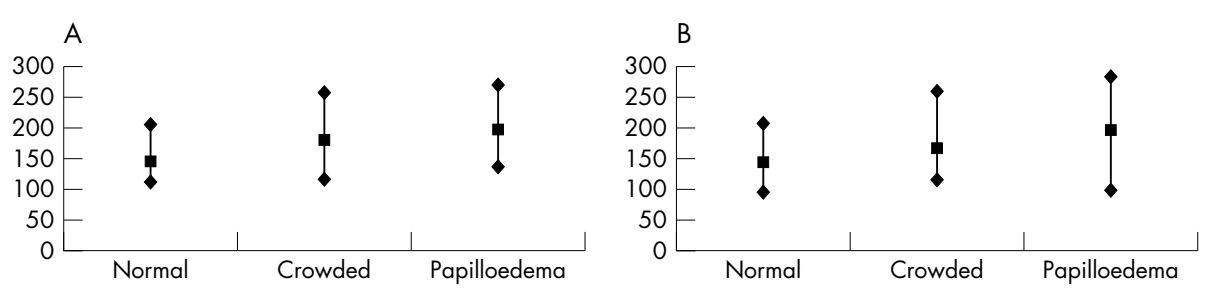

Figure 4 Distribution of nerve fibre layer thickness, average in all four quadrants in all normal, papilloedema and crowded optic discs. (A) Superior quadrant, (B) inferior quadrant, (C) nasal quadrant, (D) temporal quadrant.
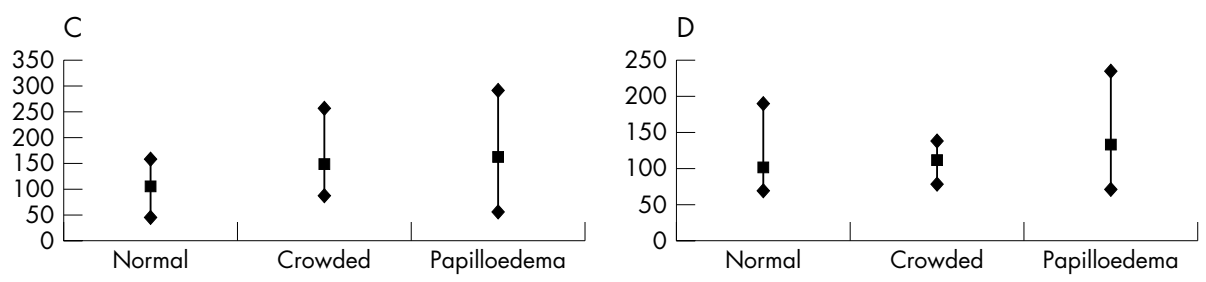

\section{DISCUSSION}

Optical coherence tomography (OCT) is a technique that provides information about tissue microstructure similar to that seen histopathologically. ${ }^{5}$ This technique has been employed in the study of different ophthalmic disorders, including glaucoma. ${ }^{7-9}$ We employed OCT to evaluate RNFL in patients with congenital crowding of the optic nerve and patients with mild papilloedema in order to determine if a distinction could be made between these two entities. The difficulty in clinically differentiating mild optic disc swelling from congenital crowding of the nerves is illustrated in the evaluation of fundus photographs by two observers in this study where there was considerable disagreement. The selection of the cases may have been biased to some degree. However the selection of papilloedema cases was limited to those with mild papilloedema. Had patients with more severe papilloedema been included, then the OCT findings may have shown thicker NFL measurements. At the same time, had patients with crowded optic discs with drusen been included OCT would have shown thinner NFL measurements. The difference between papilloedema and congenital crowding from less selective groups of patients very likely would have been present. However, many of these cases could have been easily distinguished clinically, and this was not the intention of the study. We were specifically interested in those patients in whom the difference between congenital crowding without drusen and mild papilloedema is difficult to identify clinically. We also realise that the power of the statistics used in this study is limited, and that a larger number of cases would be helpful to confirm our findings.

OCT in this study showed that the distribution of RNFL thickness around the optic disc has a typical "double hump" pattern in normal eyes, ${ }^{10}$ as well as those with congenital crowding and mild papilloedema. Patients with crowded optic nerves and mild papilloedema had statistically significant increases in mean RNFL thickness compared to normal control subjects within the study and increased RNFL thickness when compared to normal subjects from previous studies. ${ }^{6}{ }^{7}$ There were greater degrees of thickening in the superior and inferior quadrants, which is similar to what has been noted in experimental papilloedema. ${ }^{11}$ Differences in amount of glial support of RNFL bundles above and below the optic disc, as opposed to medially and temporally, may play a part in the distribution of the RNFL swelling in papilloedema just as these factors may have a role in the degeneration of axons seen in glaucoma. ${ }^{12}$

Because, in this study, mean RNFL thickness was increased in both mild papilloedema and congenital crowding of the optic nerve, a distinction between these two entities, especially on a case by case basis may not be possible by OCT alone. In this study, patients with mild papilloedema had higher values than those with congenital crowding, but this was not statistically significant. It is likely that RNFL thickness in patients with more severe papilloedema would

Table 2 Student-Newman-Keuls comparisons of nerve fibre layer thickness by quadrant in all patients and normal subjects

\begin{tabular}{|c|c|c|c|c|c|}
\hline Comparison & MD & SE & $p$ & $q$ & $\mathrm{p}<0.05$ \\
\hline \multicolumn{6}{|l|}{ Superior } \\
\hline$P \vee N$ & 51 & 5.464 & 3 & 9.334 & Yes \\
\hline$P \vee C$ & 15.4 & 6.075 & 2 & 2.535 & No \\
\hline $\begin{array}{l}\mathrm{C} v \mathrm{~N} \\
\text { Inferior }\end{array}$ & \multicolumn{2}{|c|}{ Inferior } & & 6.204 & Yes \\
\hline $\mathrm{P} v \mathrm{~N}$ & 59.1 & 7.042 & 3 & 8.393 & Yes \\
\hline$P \vee C$ & 32.4 & 7.83 & 2 & 4.138 & Yes \\
\hline$C \vee N$ & 26.7 & 7.395 & 2 & 3.61 & Yes \\
\hline \multicolumn{6}{|l|}{ Nasal } \\
\hline $\mathrm{P} \vee \mathrm{N}$ & 55.9 & 7.726 & 3 & 7.235 & Yes \\
\hline$P \vee C$ & 12.8 & 8.591 & 2 & 1.49 & No \\
\hline $\mathrm{C} v \mathrm{~N}$ & 43.1 & 8.114 & 2 & 5.312 & Yes \\
\hline \multicolumn{6}{|l|}{ Temporal } \\
\hline$P \vee N$ & 33.5 & 6.498 & 3 & 5.155 & Yes \\
\hline$P \vee C$ & 25.7 & 7.226 & 2 & 3.557 & Yes \\
\hline $\mathrm{C} v \mathrm{~N}$ & 7.8 & 6.825 & 2 & 1.143 & No \\
\hline
\end{tabular}

C, crowded; N, normal; $P$, papilloedema; $M D$, mean deviation; $S E$, standard error. Degrees of freedom: 79. 
be statistically different from those with crowded optic nerves, but, in these cases, the difference should be obvious clinically.

The finding that both crowded optic nerves and papilloedematous optic nerves showed increased thickness of the RNFL may be related to the similarity in pathogenesis of both of these entities. In both cases axonal swelling is the result of delayed axoplasmic transport. At least in optic nerves which are crowded enough to be associated with drusen, there is evidence that normal physiological damming of orthograde axoplasmic flow anterior to the lamina cribrosa is slowed even to the point of extravasation of axoplasm into perineural spaces. ${ }^{13}$ Similar, though less chronic prelaminar axoplasmic flow stasis has been demonstrated in experimental and clinical papilloedema. ${ }^{11}$

\section{Authors' affiliations}

E Z Karam, Unidad Oftalmolgica de Caracas, Venezuela T R Hedges, New England Eye Center, Tufts University School of Medicine, Boston, MA, USA

Proprietary interest: None.

Correspondence to: Thomas R Hedges III, MD, New England Eye Center, 750 Washington Street, Box 381, Boston, MA 02111, USA; thedges@lifespan.org

Accepted for publication 1 August 2004

\section{REFERENCES}

1 Walsh FB, Blair CJ. Papilledema, optic neuritis and pseudopapilledema. The use of fluorescein in differential diagnosis. Trans Am Acad Ophthalmol Otolaryngol 1969;73:914-20.

2 Hoyt WF, Knight CL. Comparison of congenital disc blurring and incipient papilledema in red-free light- a photography study. Invest Ophthalmol Vis Sci 1973;12:241-7.

3 Bynke HG, Aberg L. Differentiation of papilledema from pseudopapilledema by fluoroscein ophthalmoscopy. Acta Ophthalmol 1970;48:752.

4 Peli E, Hedges TR, Schwartz B. Computerized enhancement of retinal nerve fiber layer. Acta Ophthalmol 1986;64:113-22.

5 Fujimoto JG, Brezinski GJ, Tarney, et al. Optical biopsy and imaging using optical coherence tomography. Nat Med 1995;1:970-2.

6 Frisen L. Swelling of the optic nerve head: a staging scheme. J Neurol Neurosurg Psychiatry 1982;45:13-18.

7 Schuman JS, Hee MR, Puliafito CA, et al. Quantification of nerve fiber layer thickness in normal and glaucomatous eyes using optical coherence tomography. Arch Ophthalmol 1995;113:586-96.

8 Schuman JS, Pedut-Kloizman T, Hertzmark E, et al. Reproducibility of nerve fiber layer thickness measurements using optical coherence tomography. Ophthalmology 1996;103:1889-90.

9 Roh S, Noecker RJ, Schuman JS. Evaluation of coexisting optic nerve head drusen and glaucoma with optical coherence tomography. Ophthalmology 1997; 104:1138-44

10 Caprioli J, Ortiz-Colberg R, Miller JM, et al. Measurements of peripapillary nerve fiber layer contour in glaucoma. Am J Ophthalmol 1989; 108:404.

11 Tso MOM, Hayreh SS. Optic disc edema in raised intracranial pressure. III. A pathologic study of experimental papilledema. Arch Ophthalmol 1977:95: 1448-57.

12 Quigley HA, Addicks EM. Quantitative studies of retinal nerve fiber layer defects. Arch Ophthalmol 1982;100:807-14.

13 Spencer WH. Drusen of the optic disc and aberrant axoplasmic transport. Am J Ophthalmiol 1978;85:21-38. 